\title{
Forma e tamanho de sementes de duas variedades de abóboras durante a secagem
}

\author{
José Roberto da COSTA JÚNIOR ${ }^{1}$, Daniel Emanuel Cabral de OLIVEIRA*, \\ José Mauro Guimarães CARVALHO ${ }^{1}$, Sarah Gabrielle Sousa BUENO ${ }^{1}$, \\ Valdenice Batista FERREIRA ${ }^{1}$, Estenio Moreira ALVES ${ }^{1}$ \\ ${ }^{1}$ Instituto Federal de Educação, Ciência e Tecnologia Goiano, Iporá, GO, Brasil. \\ (Orcid: 0000-0001-9064-7040; 0000-0002-3824-994X; 0000-0002-9475-6735; 0000-0003-2375-3446; \\ 0000-0002-8291-5022; 0000-0002-2208-7384) \\ *E-mail: oliveira.d.e.c@gmail.com
}

Recebido em 28/04/2020; Aceito em 30/12/2020; Publicado em 17/02/2021.

\begin{abstract}
RESUMO: A cultura da abóbora é de grande importância para alimentação. Nos moldes da agricultura atual, a precisão de equipamentos é essencial para o êxito das atividades de pós colheita. Objetivou-se definir a forma e tamanho de duas variedades de abóboras em diferentes teores de água durante a secagem. O experimento foi desenvolvido no Instituto Federal Goiano - Campus Iporá. As sementes foram submetidas à secagem em estufa com ventilação de ar forçado a $45^{\circ} \mathrm{C}$, até atingir o teor de água de 0,0731 para cv. Rajada e 0,0711 para o AC 53 (base seca, b.s.). Foram avaliados comprimento, largura, espessura, circularidade, esfericidade, volume, diâmetro geométrico, área superficial, área projetada, relação superfície-volume e índice de contração volumétrica. A redução do teor de água proporciona a redução dos eixos ortogonais, esfericidade, volume, diâmetro geométrico, área superficial, área projetada e índice de contração volumétrica, aumento relação superfície volume durante o processo de secagem em sementes de abóbora. Nenhuma equação adequou-se aos dados de circularidade, sendo o valor médio para o AC 53 de 53,72\% e para rajada de 60,24\%. A equação que melhor representa a contração volumétrica da cv. Rajada é a equação Polinomial e para o AC 53, a de Bala \& Woods.
\end{abstract}

Palavras-chave: Secagem; propriedades físicas; cv. Rajada; cv. AC 53.

\section{Shape and size of seeds of two varieties of pumpkins during drying}

\begin{abstract}
The pumpkin culture is of great importance for food. In the mold of today's agriculture, the precision of equipment is essential for the success of post-harvest activities. The objective was to define the shape and size of two varieties of pumpkins in different water levels during drying. The experiment was developed at the Instituto Federal Goiano - Campus Iporá. The seeds were submitted to oven drying with forced air ventilation at $45^{\circ} \mathrm{C}$, until reaching a moisture content of 0.0731 for cv. Rajada and 0.0711 for AC 53 (db). Length, width, thickness, circularity, sphericity, volume, geometric diameter, surface area, projected area, surface-volume ratio and volumetric contraction index were evaluated. The reduction of the moisture content provides the reduction of the orthogonal axes, sphericity, volume, geometric diameter, surface area, projected area and volumetric contraction index, increase in the surface volume ratio during the drying process in pumpkin seeds. No equation fit the circularity data, with the mean value for AC 53 being $53.72 \%$ and for Rajada $60.24 \%$. The equation that best represents the volumetric contraction of cv. Blast is the Polynomial equation and for AC 53, Bala \& Woods.
\end{abstract}

Keywords: drying; physical properties; cv. Rajada; cv. AC 53.

\section{INTRODUÇÃO}

A espécie Curcubita moschata (abóbora), através de registros de sítios arqueológicos, está entre as espécies mais antigas cultivadas nas américas, pois fez parte assim como feijão e milho da dieta da antiga civilização Maia, datando 10.000 anos atrás, tendo como centro de origem a região central do México (RESENDE et al. 2005).

De acordo com IBGE (2006), a produção de abóboras vindas de sementes não certificadas chamadas de "crioulas" podem variar entre 70 e $87 \%$, mostrando a importância da conservação de variedades crioulas/acessos para os agricultores familiares. Mendes et al. (2017) comprovam também a capacidade fitotécnica e o potencial agronômico para uso dos acessos em sistemas produtivos. Além também da grande variabilidade e potencial para uso desses acessos em programas de melhoramento genético vegetal de cultivares adaptadas a cada região.

Para Carvalho; Nakagawa (2012), frutos recém colhidos possuem um teor de água alto nas sementes que estão na mucilagem, sendo assim inadequados para o armazenamento com segurança e necessitando, portanto, de secagem. Essa operação é necessária pois o alto teor de água das sementes, é uma das principais causas da perda de sementes durante o armazenamento, afetam também as operações de beneficiamento, dificultando muitas vezes o manejo e reduzindo a eficiência das máquinas utilizadas nos processos de beneficiamento. As sementes de abóbora têm polpa envolvente e sua extração é feita por fermentação, tratamento químico ou por ação mecânica (CARVALHO; NAKAGAWA, 2012; DALPASCUALE et al., 1983). 
Segundo Dalpascuale (1983), a definição de secagem está compreendida de acordo com o enfoque que se deseja dar. Em estudos teóricos ela pode ser definida como um processo em que há trocas simultâneas de calor e massa entre o ar do ambiente de secagem e as sementes. De modo geral a secagem é definida como a operação unitária responsável pela redução do teor de água de certos produtos até um nível considerado seguro para o armazenamento. Esse nível seguro é definido como um teor de água abaixo do qual a atividade respiratória das sementes e legumes é reduzida, e o ataque de fungos e insetos é dificultada, esse nível varia de espécie e produto agrícola, mas para condições brasileiras compreende uma faixa entre 10 a $14 \%$ de teor de água base úmida (b.u.) que é a relação do peso da água com o peso total da semente.

O processo de secagem é aplicado para reduzir o teor de água das sementes, e desse modo também é reduzido o desenvolvimento de fungos e bactérias, evitando surgimento de sementes ardidos e microtoxinas, reduzindo também as taxas respiratórias das sementes que provoca a perda de peso, e gera calor (ambiente favorável a microrganismos), e reduz a execução de reações bioquímicas que promovem a auto degeneração do produto (SILVA, 2004).

O conhecimento das características físicas de sementes tem importância fundamental na construção e operação de equipamentos de secagem e armazenagem, e também para a adaptação de equipamentos já existentes, visando obter um maior rendimento nas operações (SILVA, 2008).

Corrêa et al. (2002), cita que os eixos ortogonais de sementes de café, diminuíram com a redução do teor de água, sendo a dimensão b (largura) o eixo que mais diminuiu, influenciando diretamente na esfericidade e circularidade, indicando a geometria de um esferoide oblato a forma mais adequada para representar o fruto do café. Na mamona, a redução do teor de água, durante a secagem, promove a redução das massas específicas aparente e unitária e da porosidade dos frutos (GONELI et al., 2006).

Em cultivares de feijão a influência da secagem nas propriedades físicas foram notadas pela diminuição dos eixos ortogonais, área projetada e diâmetro geométrico. Já os valores de esfericidade e circularidade se comportaram de forma distinta (JESUS et al., 2013). Martins et al. (2017a) mostram também que a secagem causa a redução na circularidade, esfericidade e superfície projetada com a redução do teor de água, e também um aumento nos valores da relação superfície volume em sementes de açafrão.

A forma é feita por comparação com formatos padrões predeterminados, como esferas, cilindros, cones e ovalado. Projetos de máquinas para processamento, classificação e dimensionamento de equipamentos destinados à póscolheita de produtos agrícolas, requerem dados relativos às propriedades físicas, especialmente relativas às propriedades geométricas. Desta forma, objetivou-se definir a forma e tamanho de sementes de duas variedades de abóboras em diferentes teores de água durante o processo de secagem.

\section{MATERIAL E MÉTODOS}

O experimento foi realizado no Laboratório de Fitotecnia do Instituto Federal de Educação, Ciência e Tecnologia Goiano - Campus Iporá (IF Goiano - Campus Iporá). Para a condução do experimento, foram utilizadas sementes de Abóbora (Cucurbita Moschata), de duas cultivares (cv. Rajada seca melhorada e acesso 53) com teor de água de 0,5825 e 0,5783 base seca (b.s.), respectivamente.
As sementes foram retiradas e limpas da mucilagem mais grossa em processo manual, posteriormente foram colocados em uma bandeja com água para retirada da mucilagem fina, que se encontrava grudada nas sementes podendo gerar erros. Após a retirada da mucilagem as sementes foram colocadas em papel toalha para retirada do excesso de água e expostas a ambiente natural. A divisão das sementes foi feita em recipientes de alumínio, sendo colocadas uma semente em cada recipiente totalizando 15 recipientes de cada cultivar. Uma amostra foi colocada com 25 gramas de sementes para calcular o teor de água, assim foram medidos os eixos ortogonais (comprimento, largura e espessura), com o auxílio de um paquímetro digital com resolução de $0,01 \mathrm{~mm}$ (Figura1).

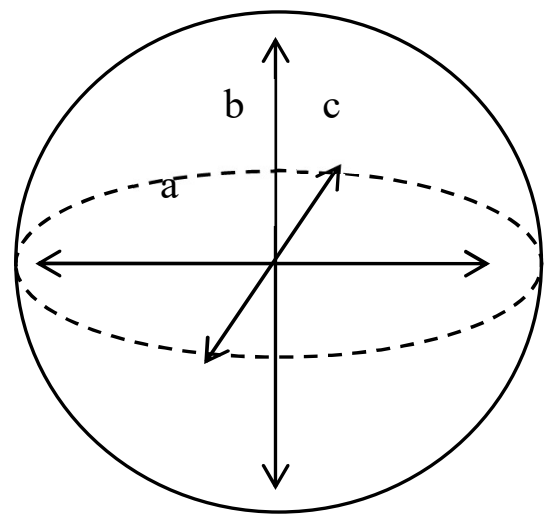

Figura 1. Desenho esquemático das sementes de abóbora (Cucurbita moschata), com suas dimensões ortogonais.

Figure 1. Schematic drawing of pumpkin seeds (Cucurbita moschata), with their orthogonal dimensions.

As sementes foram submetidas à secagem em estufa com ventilação de ar forçado na temperatura de $45^{\circ} \mathrm{C}$, até atingir o teor de água de 0,0731 para cv. Rajada e 0,0711 para o AC 53 (b.s.). Para acompanhar a perda de massa das sementes foram pesados em balança com resolução de $0,01 \mathrm{~g}$ até chegar ao teor de água desejado $(0,4453 ; 0,3300 ; 0,2317 ; 0,1469$ e 0,0731 decimal b.s.) para cv Rajada e (0,4418; 0,3270; 0,2291; 0,1447 e 0,0711 decimal b.s.) para o AC 53.

A forma das sementes, consideradas esferoides, na posição natural de repouso, foi obtida por meio da esfericidade e da circularidade de acordo com as equações 1 e 2, respectivamente, propostas por Mohsenin (1986). O volume de cada semente $\left(V_{\mathrm{s}}\right)$ foi obtido ao longo do processo de secagem de acordo com a equação 3, proposta por Mohsenin (1986). A contração volumétrica unitária $\left(\Psi_{\mathrm{u}}\right)$ foi determinada pela relação entre o volume de cada semente $\left(V_{\mathrm{s}}\right)$ para cada teor de água e o volume inicial, utilizando-se a equação 4.

O índice de contração volumétrica $\left(I \Psi_{\mathrm{u}}\right)$ foi obtido por meio da equação 5 e a área superficial (S) foi calculada pela analogia a uma esfera de mesmo diâmetro geométrico médio, por meio da equação 6, de Tunde-Akintunde; Akintunde (2004), sendo necessário para esse cálculo a determinação do diâmetro geométrico $\left(\mathrm{D}_{\mathrm{g}}\right)$, de acordo com a equação 7 , de Mohsenin (1986). A área projetada $\left(A_{p}\right)$ foi calculada de acordo com Goneli (2008) por meio da equação 8 e a relação superfície-volume (SV) por meio de a equação 9.

Aos dados experimentais do índice de contração volumétrica unitária foram ajustados os modelos matemáticos descritos pelas expressões listadas na Tabela 1. 


$$
\begin{aligned}
& \mathrm{E}_{\mathrm{S}}=\left[{\left.\frac{(\mathrm{a} \cdot \mathrm{b} \cdot \mathrm{c})^{1 / 3}}{\mathrm{a}}\right] \cdot 100}_{\mathrm{C}=\frac{\mathrm{b}}{\mathrm{a}} \cdot 100}\right. \\
& \mathrm{V}_{\mathrm{s}}=\frac{\pi \cdot \mathrm{a} \cdot \mathrm{b} \cdot \mathrm{c}}{6} \\
& \psi_{\mathrm{u}}=\frac{\mathrm{V}_{\mathrm{s}}}{\mathrm{V}_{\mathrm{s} 0}} \\
& \mathrm{I} \psi_{\mathrm{u}}=\left(\psi_{\mathrm{u} 0}-\psi_{\mathrm{ut}}\right) \cdot 100 \\
& \mathrm{~S}=\pi \cdot \mathrm{D}_{\mathrm{g}} \\
& \mathrm{D}_{\mathrm{g}}=(\mathrm{a} \cdot \mathrm{b} \cdot \mathrm{c})^{1 / 3} \\
& \mathrm{~A}_{\mathrm{p}}=\frac{\pi \mathrm{ab}}{4} \\
& \mathrm{SV}=\frac{\mathrm{S}}{\mathrm{V}_{\mathrm{f}}}
\end{aligned}
$$

em que; a: maior eixo da semente, $(\mathrm{mm})$; b: eixo médio da semente, $(\mathrm{mm})$; c: menor eixo da semente, $(\mathrm{mm}) ; \mathrm{E}_{\mathrm{s}}=$ esfericidade; $\mathrm{C}=$ circularidade; $\psi_{\mathrm{u}}=$ contração volumétrica unitária, (decimal); $\mathrm{V}_{\mathrm{s}}=$ volume da semente no tempo t, $\left(\mathrm{mm}^{3}\right) ; \mathrm{V}_{\mathrm{s} 0}=$ volume inicial $\mathrm{da}$ semente, $\left(\mathrm{mm}^{3}\right) ; \mathrm{S}=$ área superficial, $\left(\mathrm{mm}^{2}\right) ; \ddot{I} \psi_{\mathrm{u}}=$ índice de contração volumétrica, $(\%) ; \psi_{\mathrm{u} 0}=$ contração volumétrica unitária inicial, (decimal); $\psi_{\mathrm{ut}}=$ contração volumétrica unitária no tempo t, (decimal); $\mathrm{D}_{\mathrm{g}}$ : diâmetro geométrico médio, $(\mathrm{mm}) ; \mathrm{A}_{\mathrm{p}}=$ área projetada, $\left(\mathrm{mm}^{2}\right)$ e SV = relação superfície volume.

Tabela 1. Modelos utilizados para simular o índice de contração volumétrica de sementes.

Table 1. Models used to simulate the index of volumetric contraction of seeds.

\begin{tabular}{ll}
\hline \multicolumn{1}{c}{ Referência } & \multicolumn{1}{c}{ Modelo } \\
\hline $\begin{array}{l}\text { Corrêa et al. }(2002)- \\
\text { (Cor.) }\end{array}$ & $\psi=1 /(\mathrm{a}+\mathrm{b} \cdot \operatorname{Exp}(\mathrm{X}))$ \\
Exponencial $-($ Exp.) & $\psi=\mathrm{a} \cdot \operatorname{Exp}(\mathrm{b} \cdot \mathrm{X})$ \\
Linear $-($ Lin.) & $\psi=\mathrm{a}+\mathrm{b} \cdot \mathrm{X}$ \\
Polinomial - (Pol.) & $\psi=\mathrm{a}+\mathrm{b} \cdot \mathrm{X}+\mathrm{c} \cdot \mathrm{X}^{2}$ \\
$\begin{array}{l}\text { Bala \& Woods (1984) } \\
\text { modificado }\end{array}$ & $\psi=1-\mathrm{a}\left[1-\operatorname{Exp}\left[-\mathrm{b}\left(\mathrm{X}_{\mathrm{i}}-\mathrm{X}\right)\right]\right]$
\end{tabular}

em que: $\psi$ : índice de contração volumétrica; X: teor de água do produto, (\% b.s.); Xi: teor de água inicial do produto, (\% b.s.); a, b, c: coeficientes do modelo.

Para o ajuste dos modelos matemáticos, realizou-se a análise de regressão não-linear e linear pelo método GaussNewton, utilizando-se o programa statístico ${ }^{R}$. Os modelos foram selecionados, considerando-se a magnitude do coeficiente de determinação $\left(\mathrm{R}^{2}\right)$, a magnitude do erro médio relativo $(\mathrm{P})$, erro-padrão da estimativa $(\mathrm{SE})$ e o teste do Quiquadrado $\left(\chi^{2}\right)$.

$$
\begin{aligned}
& \mathrm{P}=\frac{100}{\mathrm{n}} \sum \frac{|\mathrm{Y}-\hat{\mathrm{Y}}|}{\mathrm{Y}} \\
& \mathrm{SE}=\sqrt{\frac{\sum(\mathrm{Y}-\hat{\mathrm{Y}})^{2}}{\mathrm{GLR}}} \\
& \chi^{2}=\frac{\sum(\mathrm{Y}-\hat{\mathrm{Y}})^{2}}{\mathrm{GLR}}
\end{aligned}
$$

em que: $\mathrm{Y}$ : valor observado experimentalmente; $\hat{\mathrm{Y}}$ : valor calculado pelo modelo; n: número de observações experimentais; GLR: Graus de liberdade do modelo (número de observações menos o número de parâmetros do modelo).

O experimento foi realizado com temperatura de secagem $\left(45^{\circ} \mathrm{C}\right)$ com seis teores de água $(0,5783 ; 0,4418 ; 0,3270 ; 0,2291$; 0,1447 ; 0,0711 b.s.) para o AC 53 e $(0,5825 ; 0,4453$; 0,3300 ; $0,2317 ; 0,1469 ; 0,0731)$ para Rajada, em delineamento inteiramente casualizado, com quinze repetições. Os dados foram analisados por meio de análise de variância e regressão, adotando-se o nível de 5\% de significância.

\section{RESULTADOS}

O comprimento das sementes teve uma redução de tamanho de acordo com a redução do teor de água, tendo a Rajada (3,30\%) uma perda de comprimento mais acentuada em relação ao AC 53 (1,90\%), que reduziu menos seu tamanho, mesmo tendo um comprimento maior em comparação a cv. Rajada (Figura 2A).

(A)

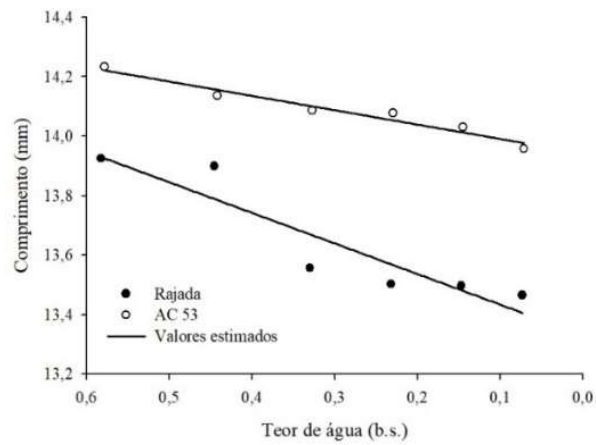

(B)

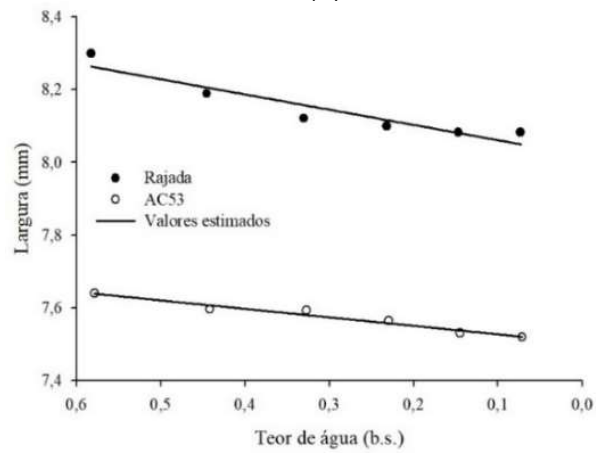

(C)

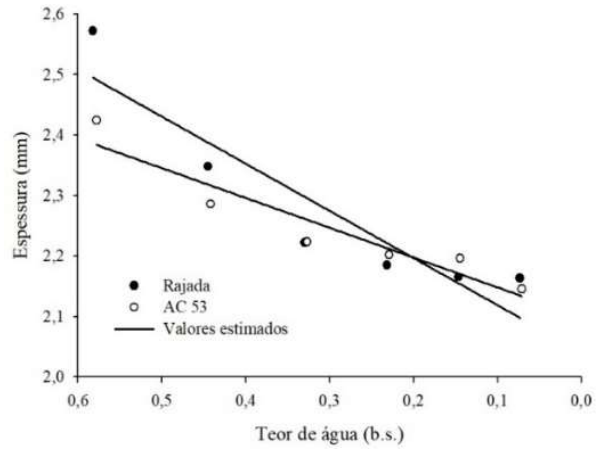

Figura 2. Comprimento (A), largura (B) e espessura (C) ( $\mathrm{mm}$ ) em função do teor de água (b.s.) das sementes de abóbora (Cucurbita moschata) da cv. Rajada e AC53.

Figure 2. Length (A), width (B) and thickness (C) (mm) as a function of the moisture content (db) of pumpkin seeds (Cucurbita moschata) from cv. Rajada and AC53. 
Já a redução da largura, devido a secagem, foi de 2,61 e $1,58 \%$, Rajada e AC53, respectivamente. Observa-se que a Rajada tem sementes mais largas em relação ao AC 53 (Figura 2B).

A esfericidade das sementes de abóbora obteve redução em relação diferente da circularidade, sendo de 3,3\% e 3,8\% do AC 53 e cv. Rajada de redução em relação ao teor de água inicial (Figura 3B).

(A)

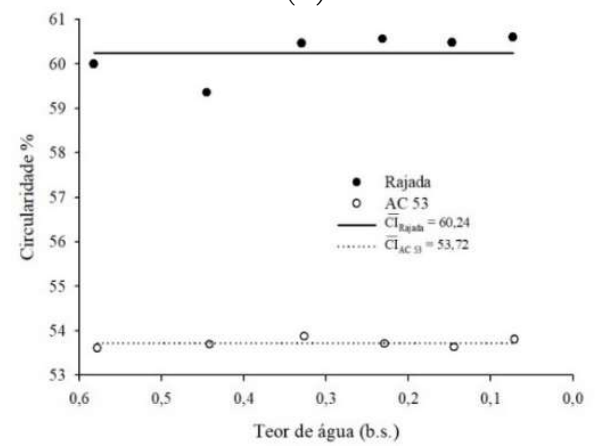

(B)

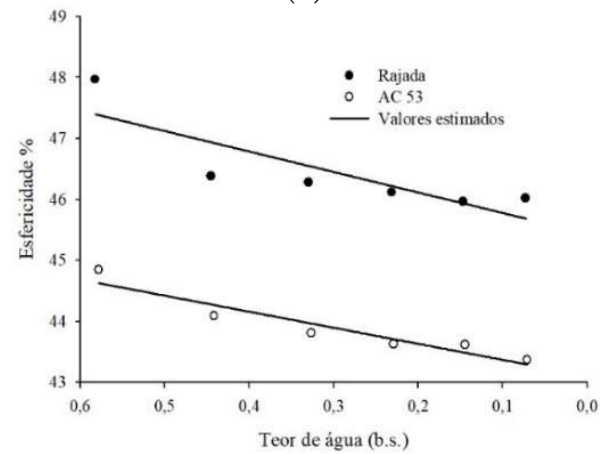

Figura 3. Circularidade (A) e esfericidade (B) (\%) em função do teor de água (b.s.) das sementes de abóbora (Cucurbita moschata) da cv. Rajada e AC53.

Figure 3. Circularity (A) and sphericity (B) (\%) as a function of the moisture content $(\mathrm{db})$ of the pumpkin seeds (Cucurbita moschata) of the cv. Rajada and AC53.

O volume (A) e o diâmetro geométrico (B) das sementes de abóbora (Cucurbita moschata), para as cultivares Rajada e AC53, em função do teor de água (base seca) podem ser observados da Figura 4.

A área superficial representada na Figura $5 \mathrm{~A}$ teve um decréscimo durante a secagem de 13,04 e 19,85 $\mathrm{mm}^{2}$ respectivamente para o AC 53 e Rajada. A área projetada (Figura 5B) diminuiu com a redução do teor de água, tendo uma perda de 3,00 e 5,02 $\mathrm{mm}^{2}$ respectivamente para o AC 53 e Rajada.

(A)

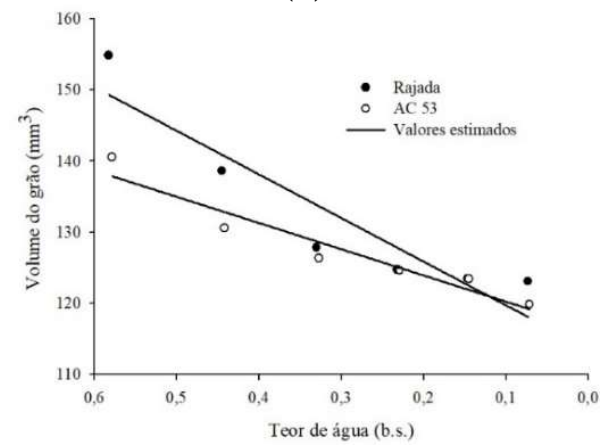

(B)

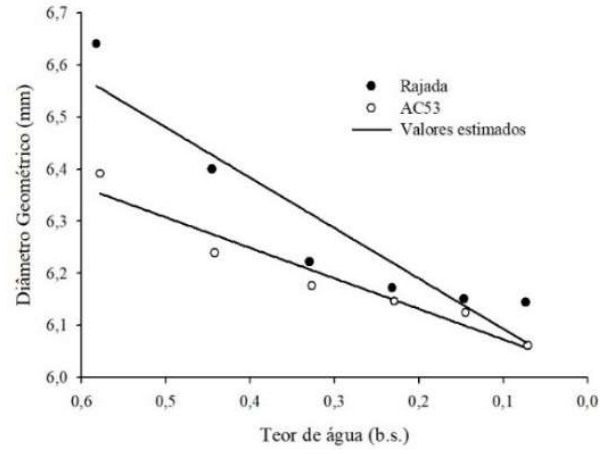

Figura 4. Volume (A) $\left(\mathrm{mm}^{3}\right)$ e diâmetro geométrico (B) $(\mathrm{mm})$ das sementes de abóbora (Cucurbita moschata) em função do teor de água (b.s.) das cv. Rajada e AC53.

Figure 4. Volume (mm3) and geometric diameter $(\mathrm{mm})$ of pumpkin seeds (Cucurbita moschata) as a function of the moisture content (db) of the cv. Rajada and AC53.

Na Figura 6, nota-se um aumento da relação superfície volume durante a secagem, tendo a cv. Rajada um maior aumento, $(7,5 \%)$ e do AC $53(5,1 \%)$.

(A)

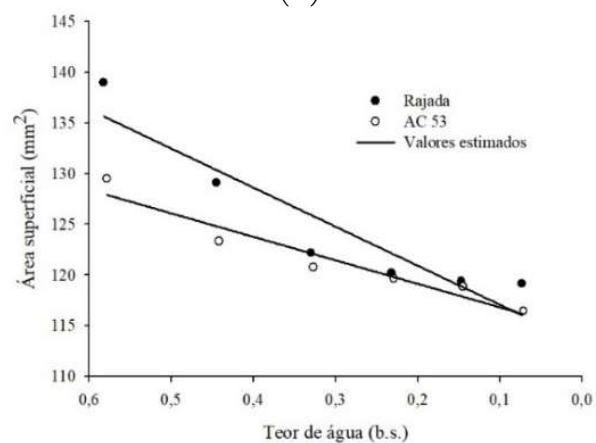

(B)

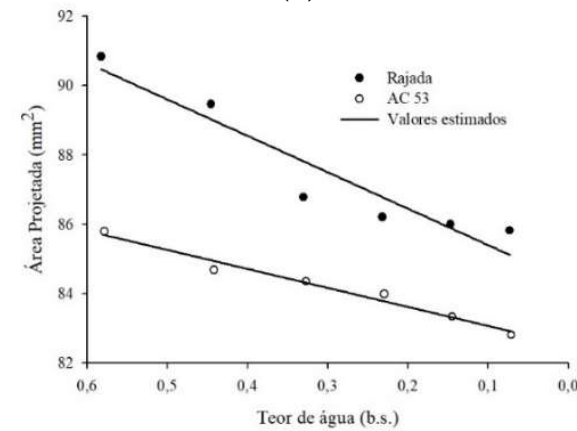

Figura 5. Área superficial (A) $\left(\mathrm{mm}^{2}\right)$ e área projetada (B) $\left(\mathrm{mm}^{2}\right)$ das sementes de abóbora (Cucurbita moschata) em função do teor de água (b.s.) das cv. Rajada e AC53.

Figure 5. Surface area (A) $\left(\mathrm{mm}^{2}\right)$ and projected area (B) $\left(\mathrm{mm}^{2}\right)$ of pumpkin seeds (Cucurbita moschata) according to the moisture content (db) of the cv. Rajada and AC53.

Os modelos apresentam altos valores do coeficiente de determinação $\left(\mathrm{R}^{2}\right)$ valores reduzidos do erro médio estimado (SE) e erro médio relativo (P) inferiores a 10\%, enfatizando que esses modelos podem representar o processo estudado (Tabela 3). Porém destaca-se os modelos Polinomial e Bala \& Woods podem representar a cv. Rajada por terem altos valores do coeficiente de determinação $\left(\mathrm{R}^{2}\right)$ acima de 0,9929 e valores reduzidos de erro médio relativo $(\mathrm{P})$ e estimado (SE), mas o modelo que melhor representa essa cultivar é o Polinomial. Para o AC 53 os modelos que podem representar são Corrêa et al., Polinomial e Bala \& Woods que possuem 
valores do coeficiente de determinação $\left(\mathrm{R}^{2}\right)$ acima de 0,9645 e valores reduzidos de erro médio relativo $(\mathrm{P})$ e estimado (SE), sendo o modelo que melhor representa o acesso é o Bala \& Woods.

A Figura 7 mostra o índice de contração volumétrica, que se reduz gradativamente com a redução do teor de água. $\mathrm{O}$ índice tem variação de $20,5 \%$ para a cv. Rajada, número superior aos $14,7 \%$ do AC 53 .

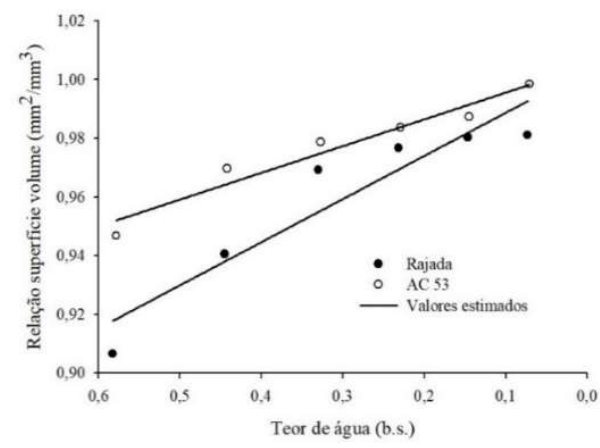

Figura 6. Relação superfície volume $\left(\mathrm{mm}^{2} / \mathrm{mm}^{3}\right)$ em função do teor de água (b.s.) das sementes de abóbora (Cucurbita moschata) da cv. Rajada e AC53.
Figure 6. Volume surface ratio $(\mathrm{mm} 2 / \mathrm{mm} 3)$ as a function of moisture content (db) of pumpkin seeds (Cucurbita moschata) from cv. Rajada and AC53.

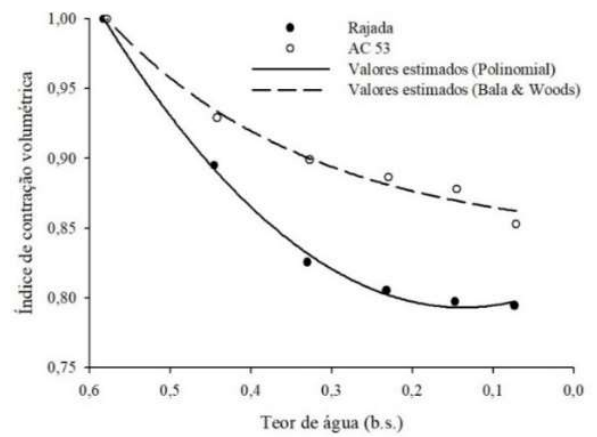

Figura 7. Índice de contração volumétrica em função do teor de água (b.s.) das sementes de abóbora (Cucurbita moschata) da cv. Rajada e AC53.

Figure 7. Volumetric contraction index a function of the moisture content (db) of pumpkin seeds (Cucurbita moschata) from cv. Rajada e AC53.

Tabela 2. Equações ajustadas aos valores do comprimento, largura, espessura, esfericidade, volume, diâmetro geométrico, área superficial, área projetada e relação superfície-volume das sementes de abóbora (Cucurbita moschata) em função do teor de água.

Table 2. Equations adjusted to the values of length, width, thickness, sphericity, volume, geometric diameter, surface area, projected area and surface-volume ratio of pumpkin seeds (Cucurbita moschata) as a function of moisture content.

\begin{tabular}{llclc}
\hline \multirow{2}{*}{ Avaliações } & \multicolumn{2}{c}{ Rajada } & AC53 \\
\cline { 2 - 5 } & \multicolumn{1}{c}{ Equação } & $\mathrm{R}^{2}$ (decimal) & Equaço & $\mathrm{R}^{2}(\mathrm{decimal})$ \\
\hline Comprimento & $\mathrm{A}=13,331+1,028 \mathrm{X}$ & 0,8517 & $\mathrm{~A}=13,943+0,482 \mathrm{X}$ & 0,9531 \\
Largura & $\mathrm{B}=8,019+0,419 \mathrm{X}$ & 0,8838 & $\mathrm{~B}=7,504+0,233 \mathrm{X}$ & 0,9616 \\
Espessura & $\mathrm{C}=2,041+0,779 \mathrm{X}$ & 0,8529 & $\mathrm{C}=2,099+0,493 \mathrm{X}$ & 0,9009 \\
Esfericidade & $\mathrm{Es}=45,444+3,358 \mathrm{X}$ & 0,7122 & $\mathrm{Es}=43,106+2,631 \mathrm{X}$ & 0,9038 \\
Volume & $\mathrm{V}_{\mathrm{g}}=113,605+61,340 \mathrm{X}$ & 0,8646 & $\mathrm{~V}_{\mathrm{g}}=116,556+36,858 \mathrm{X}$ & 0,9230 \\
Diâmetro Geométrico & $\mathrm{DG}=5,997+0,967 \mathrm{X}$ & 0,8737 & $\mathrm{DG}=6,015+0,586 \mathrm{X}$ & 0,9354 \\
Área Superficial & $\mathrm{AS}=113,234+38,497 \mathrm{X}$ & 0,8693 & $\mathrm{AS}=114,508+23,174 \mathrm{X}$ & 0,9296 \\
Área Projetada & $\mathrm{AP}=84,354+10,502 \mathrm{X}$ & 0,8993 & $\mathrm{AP}=82,526+5,481 \mathrm{X}$ & 0,9761 \\
Relação Superfície-volume & $\mathrm{SV}=1,003-0,147 \mathrm{X}$ & 0,8815 & $\mathrm{SV}=1,005-0,091 \mathrm{X}$ & 0,9439 \\
\hline
\end{tabular}

Tabela 3. Coeficientes de determinação $\mathrm{R}^{2}$ (\%), erros médio relativo P, (\%) e estimado (SDE, decimal) para os cinco modelos analisados, durante a contração volumétrica unitária das sementes de abóbora (Cucurbita moschata) da cv. Rajada e AC53.

Table 3. Coefficients of determination $\left(\mathrm{R}^{2}, \%\right)$, relative mean errors (MRE, \%) and estimated SE, (decimal) for the five models analyzed, during the unit volumetric contraction of pumpkin seeds (Cucurbita moschata) of cv. Rajada and AC53.

\begin{tabular}{lcccccc}
\hline \multirow{2}{*}{ Modelos } & \multicolumn{3}{c}{ Rajada } & \multicolumn{3}{c}{ AC53 } \\
\cline { 2 - 7 } & $\mathrm{R}^{2}$ & $\mathrm{P}$ & $\mathrm{SE}$ & $\mathrm{R}^{2}$ & $\mathrm{P}$ & $\mathrm{SE}$ \\
\hline Corrêa et al. & 0,9460 & 1,84 & 0,021 & 0,9646 & 0,82 & 0,011 \\
Exponencial & 0,8873 & 2,65 & 0,030 & 0,9320 & 1,17 & 0,015 \\
Linear & 0,8646 & 2,86 & 0,033 & 0,9230 & 1,26 & 0,016 \\
Polinomial & 0,9976 & 0,38 & 0,005 & 0,9747 & 0,79 & 0,011 \\
Bala \& Woods & 0,993 & 0,59 & 0,008 & 0,9840 & 0,55 & 0,007 \\
\hline
\end{tabular}

\section{DISCUSSÃO}

Os parâmetros de circularidade e esfericidade são de grande importância na verificação da velocidade terminal e do ângulo de repouso. Sendo assim, a razão entre a maior área projetada pela semente em repouso natural (Ap), e a área do menor círculo circunscrito (Ac) é obtida a circularidade, e a razão entre o diâmetro o maior círculo inscrito (di) e o diâmetro do menor círculo circunscrito (dc) é obtida a esfericidade (CORREIA et al., 2002). Nenhuma equação adequou-se aos dados de circularidade, sendo o valor médio para o AC 53 de $53,72 \%$ e para Rajada de 60,24\% (Figura $3 \mathrm{~A})$.
Os resultados de Resende et al. (2005), a circularidade aumenta durante o processo de secagem, para faixa de teor de água iguais as estudadas nesse trabalho, contrariando os resultados de Araújo et al. (2014), onde os valores não sofreram alteração para a cultura do amendoim durante a secagem, e mais diferentes ainda dos resultados de Goneli et al. (2011), que reduziram o valor para cultivares de mamona. Também se notou que a diferença genética das abóboras gerou sementes de tamanho muito diferentes na circularidade $(6,52 \%)$.

A esfericidade das sementes de abóbora obteve redução em relação diferente da circularidade, sendo de 3,3\% e 3,8\% 
do AC 53 e cv. Rajada de redução em relação ao teor de água inicial (Figura 3B). Essa redução se assemelha aos trabalhos de Araújo et al. (2014) e Guedes et al. (2011) que trabalharam com amendoim e soja, respectivamente, os dados colhidos com as sementes de café por Resende et al. (2005), foram diferentes, onde os valores da esfericidade aumentaram durante a secagem, e como notado anteriormente a esfericidade também teve diferença de tamanho entre as duas cultivares.

A redução do teor de água das sementes provocou uma redução do seu volume, notando que a cv. Rajada teve uma redução mais acentuada (Figura 4A). Resultados semelhantes foram observados nos trabalhos de Afonso Júnior e Corrêa (2000), com milho pipoca, também no trabalho com feijão de Resende et al. (2005), no trabalho com pistache de Razavi et al. (2007), em mamona no trabalho de Goneli (2008), com melão no trabalho de Bande et al. (2012) e com amendoim no trabalho de Araújo et al. (2014).

A Figura 4B mostra que o diâmetro geométrico também teve uma redução em relação ao processo de secagem, na AC 53 houve uma redução de $5,17 \%$, e a cv. Rajada teve uma redução de $7,47 \%$ ambas em relação ao teor de água inicial, concordando com os dados de Razavi et al. (2007) e Goneli et al. (2011). Já o trabalho de Siqueira et al. (2012) com pinhão manso mostra que não houve diferença estatística para o parâmetro diâmetro geométrico em 5 condições de ar durante a secagem. Oliveira et al. (2011), relataram que a maioria dos produtos agrícolas se contrai para todas as direções irregularmente, e o fator que mais influência sobre a taxa de dimensão é a temperatura e não a umidade relativa.

A área superficial representada na Figura $5 \mathrm{~A}$ teve um decréscimo durante a secagem de 13,04 e 19,85 $\mathrm{mm}^{2}$ respectivamente para o AC 53 e Rajada. Essa redução ocorre devido a contração volumétrica das sementes durante a secagem. Araújo et al. (2014) também relata esse fenômeno em sementes de amendoim; Guedes et al. (2011) com sementes de soja; Martins et al. (2017a) com sementes de cártamo e Siqueira et al. (2012) com pinhão manso.

A área projetada (Figura 5B) diminuiu com a redução do teor de água, tendo uma perda de 3,00 e 5,02 $\mathrm{mm}^{2}$ respectivamente para o AC 53 e Rajada. Esse comportamento se repete em trabalho de Araújo et al. (2014) com amendoim, Guedes et al. (2011) com soja; Martins et al. (2017a) com cártamo; Akintunde; Akintunde (2004) em sementes de gergelim; e Siqueira et al. (2012) com pinhão manso.

O aumento da relação superfície/volume se justifica pela redução do volume da semente ser maior que a redução da área superficial. A relação superfície/volume variou de 0,947 a $0,998 \mathrm{~mm}^{2} \mathrm{~mm}^{-3}$ para o AC 53 e de 0,907 a $0,981 \mathrm{~mm}^{2} \mathrm{~mm}$ ${ }^{3}$ para cv. Rajada. Repetindo o efeito ocorrido no trabalho de Araújo et al. (2014) com amendoim, Siqueira et al. (2012) com pinhão manso e com café na pesquisa de Botelho et al. (2016). Botelho et al. (2016) ressaltam que quanto maior a relação/superfície volume de uma semente, mais facilitadas são as transferências de calor e massa. Se fatores físicos estiverem envolvidos isoladamente, segundo Farinha (2008) a taxa de redução de água é proporcional à relação superfície/volume, resultando que seja constante a forma do produto, assim confirmando os dados obtidos onde a relação superfície/volume aumenta com a redução do tamanho da semente.

Verifica-se que os modelos apresentaram alto grau de significância de acordo com os coeficientes de determinação
$\left(\mathrm{R}^{2}\right)$ superiores à 0,7121 para a Rajada e superiores a 0,9008 para o AC 53 mostrando que todos os modelos descrevem de forma satisfatória o comportamento das variáveis analisadas. O coeficiente angular foi maior para a cultivar Rajada, mostrando que as alterações da forma e tamanho para esta cultivar durante o processo de secagem são maiores em relação a cultivar AC 53.

Para sementes de cártamo, Martins et al. (2017b) demonstra que todos esses modelos podem ser utilizados para representar o processo, sendo o Linear utilizado no trabalho devido sua simplicidade. Afonso Júnior; Corrêa (2000) também utilizaram o modelo Linear em duas variedades de milho pipoca para estimar os valores da contração volumétrica unitária. Para o pinhão manso Siqueira et al. (2012) demonstra que este fenômeno é satisfatoriamente descrito pela equação Polinomial, e que o diâmetro geométrico se reduz linearmente com a redução do teor de água, independentemente da condição de secagem. Botelho et al. (2018), demonstra que para a variedade de soja NS7901RR todos os cinco modelos podem representar a contração volumétrica aparente em diferentes temperaturas durante a secagem $\left(40,50\right.$ e $\left.60^{\circ} \mathrm{C}\right)$, mas para contração volumétrica unitária apenas os modelos Bala \& Woods e Polinomial podem representar nas três temperaturas. Já a variedade de soja TMG132RR, apenas Bala \& Woods não pode representar a contração volumétrica aparente, mas para a contração volumétrica unitária todas as equações representam as três temperaturas.

Verifica-se na Figura 7 que a maior contração acontece nos primeiros teores de secagem, pois é onde a água é retirada com maior facilidade, as moléculas que não fazem parte da composição de tecidos ou não ligadas fortemente ao produto (água livre). Em suma, quanto maior for a temperatura, mais rápido será a contração, demonstrado neste estudo por meio da Figura 7, corroborando com o estudo de Afonso Júnior e Corrêa (2000) com cultivares de milho pipoca.

\section{CONCLUSÕES}

O processo de secagem em sementes de abóbora, proporciona a redução do comprimento, largura, espessura, esfericidade, volume da semente, diâmetro geométrico, área superficial, área projetada e índice de contração volumétrica.

A secagem também proporciona o aumento da relação superfície volume durante o processo de secagem em sementes de abóbora.

Nenhuma equação adequou-se aos dados de circularidade, sendo o valor médio para o AC 53 de 53,72\% e para rajada de $60,24 \%$.

A equação que melhor representa a contração volumétrica unitária da cv. Rajada é a equação Polinomial e para o AC 53 a equação de Bala \& Woods.

A cv. Rajada que é vendida comercialmente e o acesso 53 do banco de germoplasma (BAG) do IFGoiano, não tiveram comportamento e desempenho diferentes durante $\mathrm{O}$ processo de secagem.

\section{REFERÊNCIAS}

AFONSO JÚNIOR, P. C.; CORRÊA, P. C. Cinética da contração volumétrica dos grãos de duas cultivares de milho-pipoca durante o processo de secagem. Revista Brasileira de Produtos Agroindustriais, Campina Grande, v.2, n.1, p.61-65, 2000. 
ARAÚJO, W. D.; GONELI, A. L. D.; SOUZA, C. M. A.; GONÇALVES, A. A.; VILHASANTI, H. C. B. Propriedades físicas dos grãos de amendoim durante a secagem. Revista Brasileira Engenharia Agrícola Ambiental, Campina Grande, v.18, n.3, p.279-286, 2014. DOI: https://doi.org/10.1590/S141543662014000300006

BALA, B. K.; WOODS, J. L. Simulation of deep bed malt drying. Journal Agricultural Engineering Research, v.30, n.3, p.235-244, 1984. DOI: https://doi.org/10.1016/S0021-8634(84)80024-4

BANDE, Y. M.; ADAM, N. M.; AZMI, Y.; JAMAREI, O. Moisture-dependent physical and compression properties of bitter melon (Citrullus colocynthis Lanatus) seeds. International Journal of Agricultural Research, v.7, p.243-254, 2012. DOI: 10.3923/ijar.2012.243.254

BOTELHO, F. M.; HAUTH, M. R.; HOSCHER, R. H.; BOTELHO, S. C. C. Modelagem matemática da contração volumétrica de grãos de soja durante o processo de secagem. Revista Engenharia $\mathbf{N a}$ $\begin{array}{llll}\text { Agricultura, Viçosa, } & \text { v.26, } & \text { n.1, } & \text { p.1-12, } 2018 .\end{array}$ https://doi.org/10.13083/reveng.v26i1.774

BOTELHO, F. M.; CORREA, P. C.; BOTELHO, S. C. C.; VARGAS-ELIAS, G. A.; ALMEIDA, M. D. S. D.; OLIVEIRA, G. H. H. Propriedades físicas de frutos de café robusta durante a secagem: determinação e modelagem. Coffee Science, Lavras, v.11, n.1, p.65-75, 2016.

CARVALHO, N. M.; NAKAGAWA, J. Sementes: ciência, tecnologia e produção. 5 ed. Jaboticabal: Funep, 2012. $590 \mathrm{p}$.

CORRÊA, P. C.; AFONSO JÚNIOR, P. C.; QUEIROZ, D. M.; SAMPAIO, C. P.; CARDOSO, J. B. Variation of characteristic dimensions and forms of coffee fruits during drying process. Revista Brasileira de Engenharia Agrícola e Ambiental, Campina Grande, v.6, n.3, p.466-460, 2002. DOI: 10.1590/S141543662002000300014

DALPASCUALE, V. A., SILVA, J. de S. Secagem de grãos. Informe Agropecuário, Belo Horizonte, v.9, n.99, p.811, 1983.

FARINHA, L. R. L. Características físicas na perda da matéria fresca e evolução das propriedades reológicas e cor da cenoura cv. Brasília no armazenamento. 2008. $111 \mathrm{f}$. (Dissertação de mestrado) - Universidade Federal de Viçosa, Viçosa, 2008.

GONELI, A. L. D. Variação das propriedades físicomecânicas e da qualidade da mamona (Ricinus Communis L.) durante a secagem e o armazenamento. 2008. 199p. Tese (Pós-Graduação em Engenharia Agrícola) - Universidade Federal de Viçosa, Minas Gerais.

GONELI, A. L. D.; CORRÊA, P. C.; RESENDE, O.; BOTELHO, F. M. Propriedades físicas dos frutos de mamona durante a secagem. Revista Brasileira de Armazenamento, Viçosa, v. 33, n. 1,p. 148-155, 2006.

GONELI, A.L.D.; CORRÊA, P.C.; MAGALHÃES, F.E.A.; BAPTESTINI, F.M. Contração volumétrica e forma dos frutos de mamona durante a secagem. Acta Scientiarum. Agronomy, Maringá, v.33, n.1, p.1-8, 2011. DOI: 10.4025 /actasciagron.v33i1.4629

GUEDES, M. A.; MATA, M. E. R. M. C.; DUARTE, M. E. M.; FARIAS, P. A.; NÓBREGA, A. M. M. C. Caracterização física de grãos de soja utilizando processamento digital de imagens. Revista Brasileira de Produtos Agroindustriais, Campina Grande, v.13, p.277-292, 2011. 8595/rbpa.v13n3p279-294

IBGE - Instituto Brasileiro de Geografia e Estatística. Banco de dados agregados: Agricultura. Rio de Janeiro, 2006. Disponível em: <http://www.sidra.ibge.gov.br/bda/tabela/listabl1.aspc $=822 \& \mathrm{n}=0 \& \mathrm{u}=0 \& \mathrm{z}=\mathrm{t} \& \mathrm{o}=11 \& \mathrm{i}=\mathrm{P}>$. Acesso em: 14/04/2018.

JESUS, F. F.; SOUZA, R. T. G.; TEIXEIRA, G. C. S.; TEIXEIRA, E. R. T.; DEVILLA, I. A. D. Propriedades físicas de sementes de feijão em função de teores de água. Revista de Engenharia na Agricultura, Viçosa, v.21, n.1, p.225-230, 2013. DOI: 10.13083/reveng.v21i1.390

MARTINS, E. A. S.; GONELI, A. L. D.; FILHO, C. P. H.; MAUAD, M.; SIQUEIRA, V. C.; GONÇALVES, A. A. Physical properties of safflower grains. Part I: Geometric and gravimetric characteristics. Revista Brasileira de Engenharia Agrícola e Ambiental, Campina Grande, v.21, n.5, p.344-349, 2017a. DOI: 10.1590/18071929/agriambi.v21n5p344-349

MARTINS, E. A. S.; GONELI, A. L. D.; GONÇALVES, A. A.; FILHO, C. P. H.; RECH, J.; OBA, G. C. Physical properties of safflower grains. Part II: Volumetric shrinkage. Revista Brasileira de Engenharia Agrícola e Ambiental, Campina Grande, v.21, n.5, p.350-355, 2017b. DOI: 10.1590/1807-1929/agriambi.v21 n5p350355

MENDES, I. B.; SANTOS, L. J.; SOUZA, S. R.; BATISTA, G. S.; CALGARO JUNIOR, G.; CUSTÓDIO A. M.; SANTOS, L. C.; PAIM, T. P.; ALVES, E. M. Desempenho e características de acessos de abóboras (Cucurbita moschata). Revista Eletrônica Interdisciplinar, Barra do Garças, v.1, n.1, p.176-181, 2017.

MOHSEnIN, N. N. Physical Properties of Plant and Animal Materials: Structure, Physical Characteristics, and Mechanical Properties https://www.google.com.br/search?hl=pt-

$\mathrm{BR} \& \mathrm{tbo}=\mathrm{p} \& \mathrm{tbm}=\mathrm{bks} \& \mathrm{q}=$ bibliogroup: $\% 22$ Structure,+ Physical+Characteristics, +and+Mechanical+Properti $\% 2$ $2 \&$ source $=$ gbs_metadata_r\&cad $=5$. Gordon and Breach, $2^{\circ}$ edição, 1986. 891p.

OLIVEIRA, J. A.; SILVA, T. T. A.; VON PINHO, E. V. R.; ABREU, L. A. S. Secagem e armazenamento de sementes de sorgo com alto e baixo teor de tanino. Revista Brasileira de Sementes, Londrina, v.33, n.4, p.699-710, 2011. DOI: $10.1590 /$ S0101-31222011000400012

RAZAVI, S. M. A.; EMADZADEH, B.; RAFE, A.; AMINI, A. M. The physical properties of pistachio nut and kernel as a function of moisture content and variety: Part I. Geometrical properties. Journal of Food Engineering, v.81, n.1, p.209-217, 2007. DOI: 10.1016/j.jfoodeng.2006.11.003

RESENDE, O.; CORREAA, P. C.; GONELI, A. L. D.; RIBEIRO, D. M. Forma, tamanho e contração volumétrica do feijão (Phaseolus vulgaris L.) durande a secagem. Revista Brasileira de Produtos Agroindustriais, Campina Grande, v.7, n.1, p.15-24, 2005.

RESENDE, P. C. Olericultura: teoria e prática. Viçosa: Editora Fontes, 2005. 486p. 
SILVA, J. S. Secagem e armazenagem de produtos agrícolas. Viçosa: Aprenda Fácil, 2008. 502 p.

SILVA, L. C. Secagem de grãos. Grãos Brasil: da semente ao consumo, Florianópolis, v.3, n.14, p.10-14, 2004.

SIQUEIRA, V. C.; RESENDE, O.; CHAVES, T. H.; SOARES, F. A. L. Forma e tamanho dos frutos de pinhão-manso durante a secagem em cinco condições de ar. Revista Brasileira de Engenharia Agrícola e Ambiental, Campina Grande, v.16, n.8, p.864-870, 2012. DOI: $10.1590 / \mathrm{S} 1415-43662012000800008$

TUNDE-AKINNTUNDE, T. Y.; AKINTUNDE, B. O. Some physical properties of sesame seed. Biosystems Engineering, v.88, n.1, p.127-129, 2004. DOI: 10.1016/j.biosystemseng.2004.01.009 